\title{
Characterization of Loss and Bandwidth Performance of Reflectarray Antenna Based on Lumped Components
}

\author{
Muhammad Yusof Ismail ${ }^{1}$, Muhammad Inam Abbasi ${ }^{2}$, Ahmad Faizal Mohd. Zain ${ }^{3}$, \\ Muhammad Amin, and Muhammad Faiz Liew Abdullah ${ }^{5}$ \\ ${ }^{1.2 .3 .5}$ Wireless and Radio Science Center (WARAS), \\ Faculty of Electrical \& Electronic Engineering \\ Universiti Tun Hussein Onn Malaysia \\ 86400 Parit Raja, Johor, Malaysia \\ ${ }^{4}$ Space and Upper Atmosphere Research Commission (SUPARCO), \\ Islamabad, Pakistan \\ yusofi@uthm.edu.my,muhammad_inamabbasi@yahoo.com,dr_faizal@yahoo.com, \\ muhammad_amin_mughal@yahoo.com,faiz@uthm.edu.my.
}

\begin{abstract}
Lumped components are used to represent the reflectarrays designed using different commercially available materials. The loss performance and the effect of material properties on the reflectarray antennas are discussed in terms of the lumped components which are used in the equivalent circuit analysis. The bandwidth performance of reflectarrays designed with different materials is discussed using reflection loss and reflection phase plots obtained by equivalent circuit analysis. Furthermore the results obtained by equivalent circuit modeling are compared with the results obtained using CST Microwave Studio simulations and a close agreement between all the results has been demonstrated. The dielectric permittivity $\left(\varepsilon_{\mathrm{r}}\right)$ of materials investigated in this work ranges between 2.08 to 13 and the loss tangent $(\tan \delta)$ values vary from 0.0003 to 0.025 while the reflection loss values obtained by equivalent circuit analysis varied from $0.179 \mathrm{~dB}$ to $6.875 \mathrm{~dB}$ and a variation in $10 \%$ and $20 \%$ bandwidth is observed from $84 \mathrm{MHz}$ to $360 \mathrm{MHZ}$ and $126 \mathrm{MHZ}$ to $540 \mathrm{MHz}$ respectively based on the respective material properties.
\end{abstract}

Keywords: Reflectarray, Lumped components, equivalent circuit analysis, Reflection loss, Bandwidth

\section{Introduction}

Reflectarray is a combination of a flat reflector and an array of microstrip patch elements printed on a thin dielectric substrate. It is illuminated using a primary feed horn placed at a particular distance from the periodic array of microstrip patch elements. The individual elements of the array are designed to scatter the incident field with proper phase distribution to form a planer phase surface in front of the array aperture [1]. The design techniques of the array element include identical patches of variable length stubs [2], square patches of variable sizes [3], and identical planar elements of variable rotation angle [4] have been widely used in order to control the phase distribution of the reflectarray antenna. The use of the reflectarray is preferred due to its significant advantages over conventional parabolic and phased array antennas. Some of the advantages are easy deployability, lower manufacturing cost, scannable beam and it is surface mountable with lower mass and volume [5]. Despite of the advantages, the use of reflectarray is limited to only few applications because of its narrow bandwidth and loss performance as compared to its conventional counterparts [6]. Many techniques have been proposed for the optimization of bandwidth and loss performance and different methods have been used to analyze the reflectarrays. This paper proposes the lumped components equivalent circuit representation of reflectarrays, designed with different commercially available

Received: February 1,2010. Accepted: May 19, 2010 
materials, which can be used for the analysis of bandwidth and loss performance. The effect of variation in the value of each lumped component on the performance of reflectarray is discussed in detail.

\section{Lumped Component Representation}

A. Theoretical Investiagation

Lumped components can be used for the equivalent circuit representation of the reflectarrays. In a reflectarray the dielectric and conductor losses are the most significant sources of losses especially at millimeter wave frequencies [1]. Dielectric losses occur due to high electric field generated in the substrate region and conductor losses occur due to high current distribution on the surface of the patch. A lossless reflectarray can be represented by a parallel LC circuit (LC resonant tank) and when the losses are considered, an additional resistor in series to the capacitor must be included in the circuit [7]. A lumped component representation of the lossy reflectarray is shown in Figure 1 where a network analyzer is connected with the equivalent circuit of reflectarray for scattering parameters measurements.

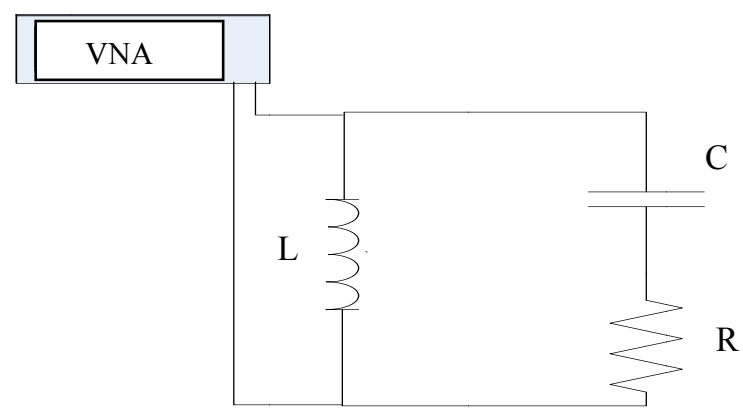

Figure 1. Equivalent circuit Model for a typical reflectarray

The capacitive losses in the reflectarray can be explained using a simple capacitor that consists of two parallel conductive plates of area A, separated by a dielectric with height $d$ and permittivity $\varepsilon$ as shown in Figure 2. The plates are considered to extend uniformly over an area A and a charge density $\pm \rho= \pm \mathrm{Q} / \mathrm{A}$ exists on their surface. Assuming that the width of the plates is much greater than their separation $d$, the electric field near the centre of the device will be uniform with the magnitude $\mathrm{E}=\rho / \varepsilon$. This assumption is also valid for every single element of a reflectarray where, the width of the patch element is kept much greater than the height of substrate. The capacitance of an ideal capacitor can be given by the ratio of the charge Q on the conducting plates to the voltage between them.

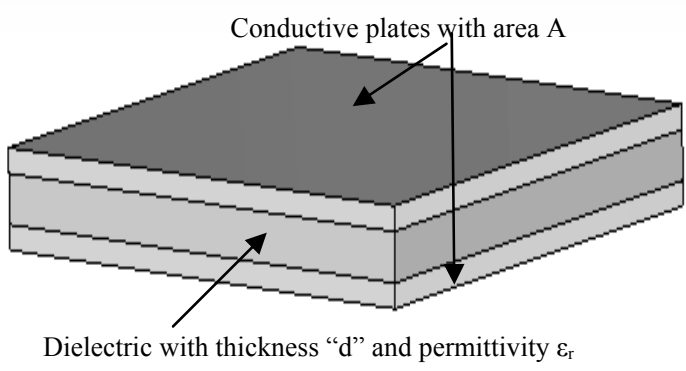

Figure 2. Structure of a simple parallel plate capacitor

$$
C=\frac{Q}{V}
$$


The voltage is defined as the line integral of the electric field between the plates

$$
V=\int_{0}^{d} E d z=\int_{0}^{d} \frac{\rho}{\varepsilon} d z
$$

Solving above integral and substituting $\rho=\mathrm{Q} / \mathrm{A}$, equation (2) becomes,

$$
V=\frac{\rho d}{\varepsilon}=\frac{Q d}{\varepsilon A}
$$

Solving this for $\mathrm{C}=\mathrm{Q} / \mathrm{V}$ reveals that capacitance increases with area and decreases with separation between the plates.

$$
C=\frac{\varepsilon A}{d}
$$

The capacitance is therefore highest in devices made from materials with a high permittivity. It can also be observed that increasing the distance $\mathrm{d}$ between the plates, which is equivalent to increasing substrate thickness in the reflectarray, has the same effect as decreasing the permittivity $\varepsilon$. This reduces the capacitive effect of the capacitor. Hence reducing the dielectric absorption in the dielectric layer and causing a drop in the losses of reflectarray. Therefore in order to improve the performance of the reflectarray antenna the capacitance should be smallest for the reflectarray designed with a material having lowest permittivity. As mentioned earlier the resistor in the series of the capacitor is added for the introduction of losses in the reflectarray, the resistance of the lumped component representation of the reflectarray designed with highest loss tangent $(\tan \delta)$ value should be highest. The capacitance given in equation (3) is for a theoretically ideal capacitor. But in real, a capacitor consists of an ideal capacitor $C_{i d e a l}$, a parallel resistance $R_{p}(E P R)$, an equivalent series resistance $R_{s}(E S R)$ and an equivalent series inductance $L_{s}(E S L)$ [8] as shown in Figure 3.

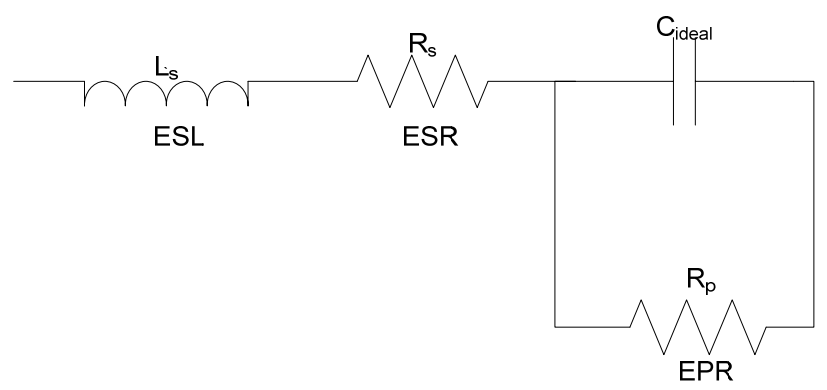

Figure 3. Equivalent circuit for a real capacitor (Lossy capacitor)

The circuit in Figure 3 indicates that every capacitor has a self-resonant frequency, above which it becomes an inductor. ESR is readily measured by applying this frequency to a capacitor, measuring the voltage and current, and calculating the ratio. The capacitive and inductive reactances cancel at the resonant frequency, leaving only ESR to limit the current. The resistance EPR will always be much larger than the capacitive reactance at the resonant frequency, so this resistance can be neglected for this computation and the equivalent circuit of a real capacitor can be given as in Figure 4. The ESR represents losses in the capacitor [8] and can be given by:

$$
E S R=\frac{\sigma}{\varepsilon^{\prime} \omega^{2} C}
$$

Where, $\sigma$ is the conductivity of the material and $\varepsilon^{\prime}$ is the real part of the dielectric permittivity of the substrate. $C$ represents the ideal or lossless capacitance. In a low-loss capacitor the ESR is very small, and in a lossy capacitor the ESR can be large. When 
representing the electrical circuit parameters as vectors in a complex plane, known as phasors, a capacitor's loss tangent is equal to the tangent of the angle between the capacitor's impedance vector and the negative reactive axis [8], as shown in the Figure 4. The loss tangent can then be given by:

$$
\tan \delta=\frac{E S R}{|X c|}=\omega C \cdot E S R=\frac{\sigma}{\varepsilon^{\prime} \omega}
$$
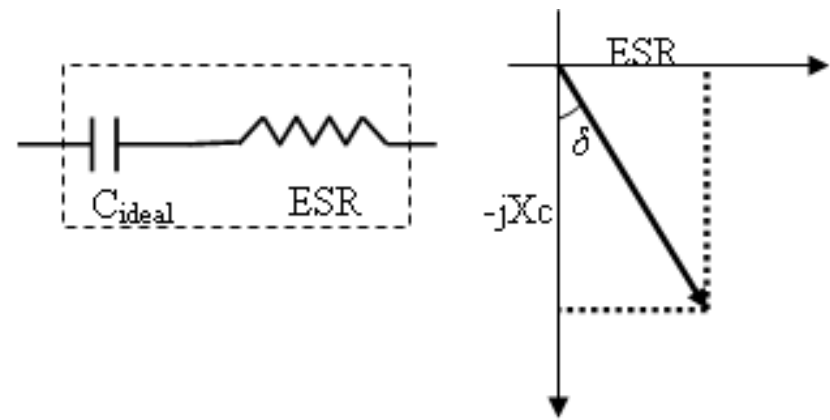

Figure 4. Real Capacitor and the loss tangent shown in impedance plane

From equation (4) and equation (5), it can be seen that the series resistance which represents the losses in a reflectarray is dependent on the dielectric properties of the substrate used for the design. Moreover it can also be observed that ESR decreases with the decrease in the loss tangent value of the substrate. Therefore a reflectarray antenna designed with low loss tangent material exhibits lower reflection loss.

Every inductor has a certain amount of resistance which causes a loss in the form of heat and is represented by copper loss. Normally this resistance is small which can be neglected in solving various types of circuit problems because the reactance of the inductor (the opposition to alternating current) is much greater than the resistance. Therefore the resistance has a negligible effect on the current and the loss due to inductance in a reflectarray is very low. However the value of the inductance is important for the calculation of resonant frequency of the reflectarrays. The resonant frequency of an equivalent LC circuit of a reflectarray antenna is given by:

$$
f_{r}=\frac{1}{2 \pi \sqrt{L C}}
$$

Different combination values of inductance (L) and capacitance (C) for one resonant frequency can be used to model the loss performance of a reflectarray antenna. High values of inductance $L$ with low capacitance $C$, result in an increase in the respective reactances $X_{L}$ and $X_{C}$. This will cause a small amount of currents circulating in the LC tank. As a result, energy lost in resistance $\mathrm{R}$ will be smaller and hence causing low loss performance of the circuit. In order to optimize the loss performance of a reflectarray antenna, the value of $\mathrm{L}$ should be maximum with a minimum value of $\mathrm{C}$ for the material.

\section{B. Equivalent circuit of reflectarrays:}

For the validation of this theory, reflectarrays printed above different materials were designed and simulated with lumped components using commercially available computer model of MUTISIM ${ }^{\mathrm{V} 10}$. The values of the lumped components can be calculated by the following relationships [7].

$$
C=\frac{\sigma_{r}}{8 \pi \eta_{r}}
$$




$$
L=\frac{2 \eta_{r}}{\pi f_{r} \sigma_{r}}
$$

Where, $\eta_{r}=377 \Omega$ is the characteristics impedance of vacuum and $\sigma_{r}$ is the phase derivative at $f_{r}$. The value of the resistor can be approximately calculated by:

$$
R \approx \frac{1-\Gamma_{r}}{1+\Gamma_{r}} \frac{16 \eta_{r}}{f_{r}^{2} \sigma_{r}^{2}}
$$

Where, $\Gamma_{r}$ is the reflection coefficient at the resonant frequency $f_{r}$. The above equations are used for the calculation of the values of R, L and C for different materials used in the design of reflectarrays as given in Table 1 .

Table 1. Lumped Component Values For Reflectarrays Of Different Materials

\begin{tabular}{cccccccc}
\hline Material & $\boldsymbol{\varepsilon}_{\mathbf{r}}$ & Tan $\boldsymbol{c}$ & $\begin{array}{c}\text { Reflection } \\
\text { loss }(\mathbf{R I}) \\
(\mathbf{d B})\end{array}$ & $\begin{array}{c}\boldsymbol{\sigma}_{\mathbf{r}} \\
(\mathbf{r a d} / \mathbf{G H z})\end{array}$ & $\begin{array}{c}\mathbf{R} \\
(\mathbf{m} \mathbf{\Omega})\end{array}$ & $\begin{array}{c}\mathbf{L} \\
(\mathbf{n H})\end{array}$ & $\begin{array}{c}\mathbf{C} \\
(\mathbf{p F})\end{array}$ \\
\hline Teflon & 2.08 & 0.0004 & 0.179 & 3.647 & 46.7 & 0.65 & 0.38 \\
Vaseline & 2.16 & 0.001 & 0.261 & 3.752 & 64.4 & 0.64 & 0.396 \\
Roger 5880 & 2.2 & 0.0004 & 0.180 & 3.805 & 43.2 & 0.63 & 0.401 \\
Roger 5870 & 2.33 & 0.0012 & 0.313 & 3.979 & 68.6 & 0.60 & 0.42 \\
CEM & 4.5 & 0.025 & 6.875 & 6.7370 & 500.1 & 0.35 & 0.71 \\
Beryllia & 6.5 & 0.0004 & 0.395 & 9.976 & 21.6 & 0.30 & 0.84 \\
Alumina 95\% & 9.75 & 0.0003 & 0.519 & 10.14 & 17.5 & 0.236 & 1.1 \\
Silicon & 11.9 & 0.004 & 2.857 & 12.47 & 63.1 & 0.19 & 1.3 \\
Gallium Arsenide & 13 & 0.006 & 4.326 & 13.24 & 83.9 & 0.18 & 1.4 \\
\hline
\end{tabular}

It can be observed from Table 1 that reflectarray printed above dielectric material of Teflon with the lowest value of $\mathrm{C}=0.38 \mathrm{pF}$ and highest value of $\mathrm{L}=0.65 \mathrm{nH}$ offers a reflection loss of $0.179 \mathrm{~dB}$ for the equivalent circuit representation. However it is shown that reflectarray constructed above Gallium Arsenide with the highest value of $\mathrm{C}=1.4 \mathrm{pF}$ and lowest value of $\mathrm{L}=0.18 \mathrm{nH}$ contributes higher reflection loss of $4.326 \mathrm{~dB}$ compared to Teflon. This is in good agreement with the effect explained above. However in order to measure effective reflection loss performance, the value of the resistor must also be taken into consideration. As shown in Table1, it can also be observed that the loss tangent value (tan $\delta)$ of CEM is the highest among the available materials causing the value of resistance $\mathrm{R}$ to be the highest for CEM. This is the reason CEM is showing maximum value of reflection loss (Rl). If Teflon and Beryllia are compared, it can be observed that both have the same value of loss tangent $(\tan \delta=0.0004)$, but Beryllia has a reflection loss value of $0.395 \mathrm{~dB}$ as compared to the Teflon which has a reflection loss of $0.179 \mathrm{~dB}$. This is because of the difference in the material properties where Teflon has a permittivity $\varepsilon_{\mathrm{r}}=2.08$ and Beryllia has a dielectric constant $\varepsilon_{\mathrm{r}}=6.5$. So for Teflon the value of the capacitance is lower $(\mathrm{C}=0.38 \mathrm{pF})$ as compared to Beryllia $(\mathrm{C}=0.84 \mathrm{pF})$ and the value of inductance for Teflon $(\mathrm{L}=0.65 \mathrm{nH})$ is higher than Beryllia $(\mathrm{L}=0.30 \mathrm{nH})$. Therefore the reactance $X_{L}$ and $X_{C}$ will be lower for Teflon as compared to Beryllia. Due to the lump components effect, the reflectarray design with Beryllia shows more losses compared to reflectarray antenna printed above Teflon. 


\section{Simulations and comparisons}

The reflection loss curves and reflection phase curves for Teflon obtained by equivalent circuit modeling using MULTISIM ${ }^{\mathrm{V} 10}$ are compared with the results obtained by CST Microwave Studio (CST MWS) as shown in Figure 5.

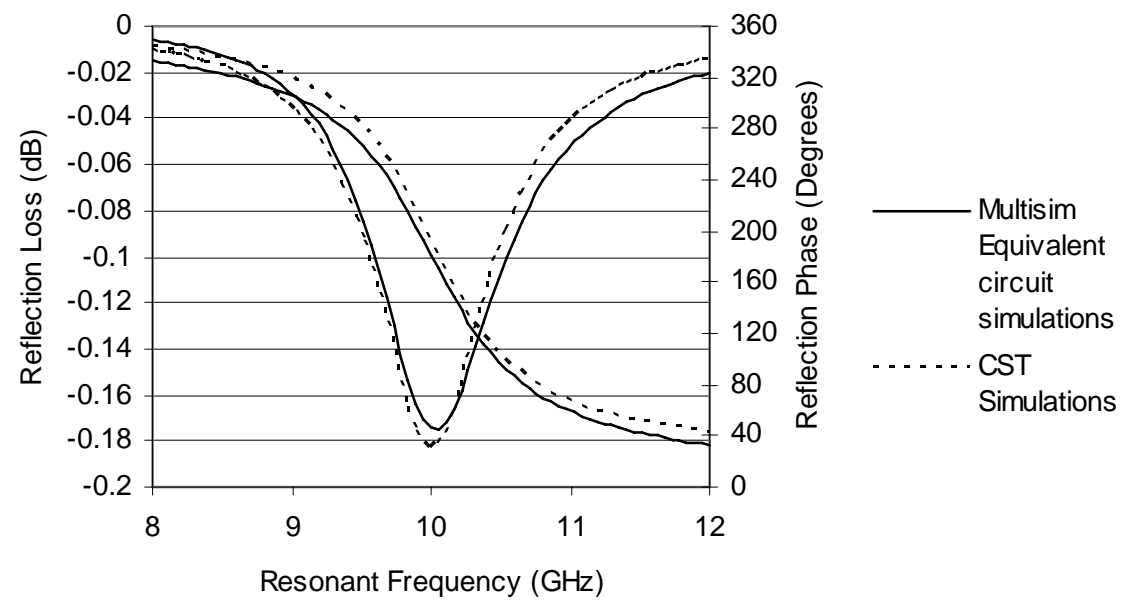

Figure 5. Comparison of CST simulations with equivalent circuit analysis for Teflon

It can be observed from Figure 5 that the results produced by equivalent circuit modeling are in good agreement with the CST Microwave Simulated results. Furthermore it can be observed from Figure 5 that Teflon gives out a very low loss of $0.17 \mathrm{~dB}$. On the other hand the slope of the reflection phase curve which, is a measure of reflectarray bandwidth [1], for Teflon is very smooth which shows that Teflon shows a better bandwidth performance when used for the design of a reflectarray.

Figure 6 shows a comparison of CST MWS and equivalent circuit modeling results. It can be observed from Figure 6 that both the computer models produced almost identical results. When a reflectarray is designed with Gallium Arsenide at $10 \mathrm{GHz}$, it gives a reflection loss of $4.3 \mathrm{~dB}$ and a very steep slope of the reflection phase curve. This is because of the fact that Gallium Arsenide has a ver high loss tangent value of 0.006 and a very high value of dielectric constant $\left(\varepsilon_{\mathrm{r}}=13\right)$.

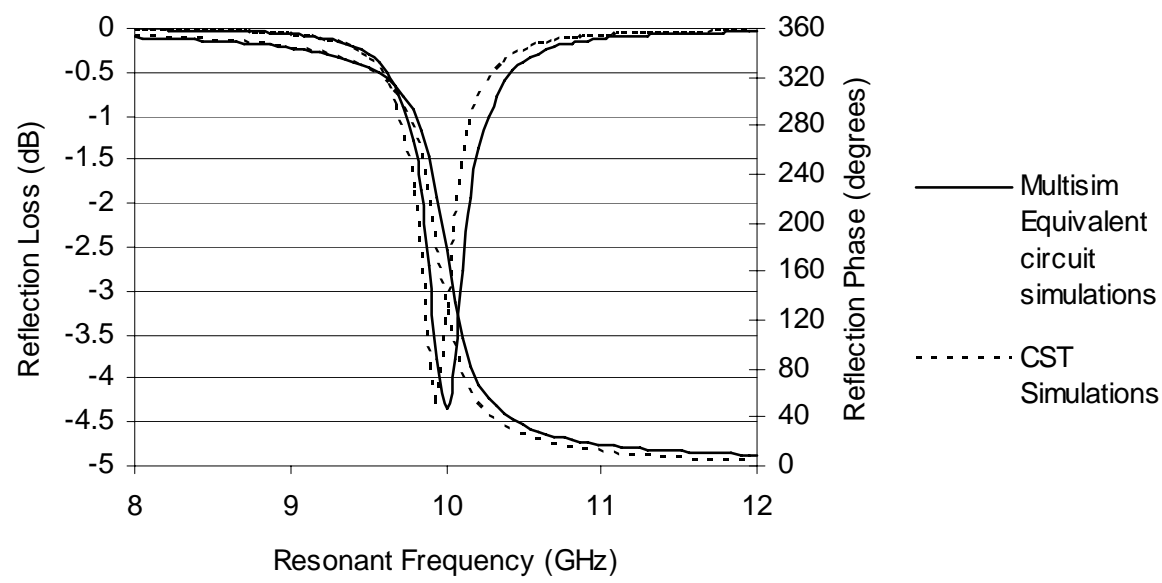

Figure 6. Comparison of CST simulations with equivalent circuit analysis for Gallium Arsenide 


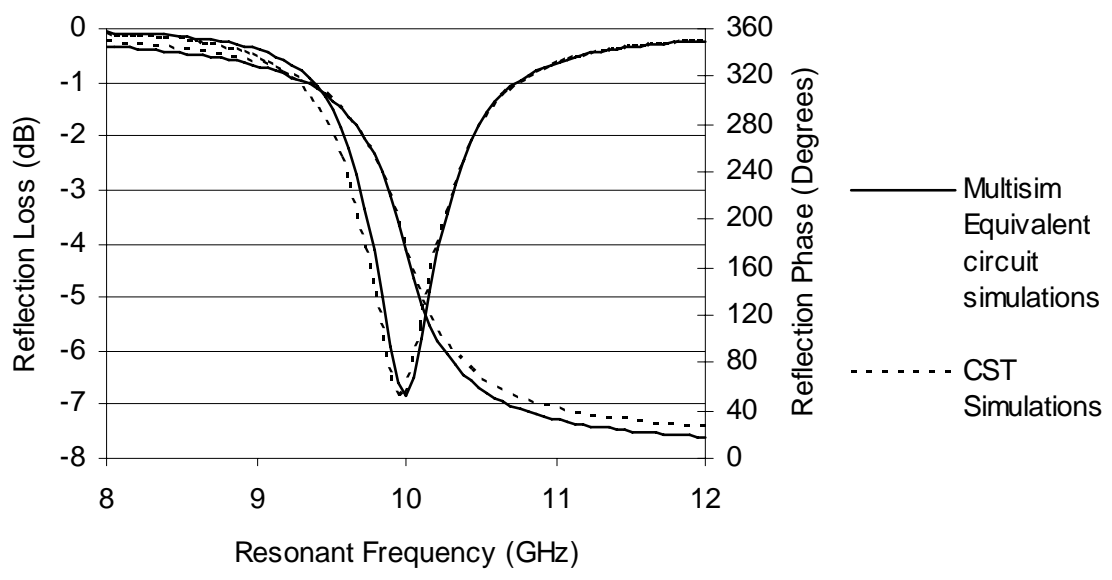

Figure 7. Comparison of CST simulations with equivalent circuit analysis for CEM

The comparison between the equivalent circuit modeling results and the results obtained from CST MWS for the reflectarray designed with CEM as substrate is shown in Figure 7. A close agreement between the two set of results can be observed from Figure 7. Furthermore a high reflection loss of $6.8 \mathrm{~dB}$ is observed for reflectarray designed with CEM. However the slope of the phase is less steep as compared to Gallium Arsenide. This is because of the fact that CEM has a very high loss tangent value of 0.025 which makes it a high loss material. On the other hand the dielectric constant for CEM is 4.5 as compared to 13 for Gallium Arsenide which causes smoother slope of reflection phase curve and hence more bandwidth. The bandwidth performance of reflectarray design with different materials calculated at different levels is shown in Table 2. The $10 \%$ and $20 \%$ bandwidths as shown in Table 2 are calculated by moving $10 \%$ and $20 \%$ above the reflection loss value at resonant frequency for comparison. Although the reflection loss value at resonant frequency of $10 \mathrm{GHz}$ for CEM is much higher than that of Gallium Arsenide, but the bandwidth performance of reflectarray designed with CEM is still better than Gallium arsenide. This is because of the fact that as the bandwidth of reflectarray is also inversely proportional to the dielectric permittivity of the material used for design and it can be improved by using thicker substrate which has the same effect as the reducing the values of dielectric permittivity. This fact can also be demonstrated by comparing equation (3) and equation (6), which is used for the calculation of lumped component values.

Table 2. Bandwidths For Different Materials

\begin{tabular}{lcc}
\hline \multicolumn{1}{c}{ Dielectric Material } & $\begin{array}{c}\mathbf{1 0 \%} \text { Bandwidth } \\
\text { (MHz) }\end{array}$ & $\begin{array}{c}\mathbf{2 0 \%} \text { Bandwidth } \\
\text { (MHz) }\end{array}$ \\
\hline Teflon & $360 \mathrm{MHz}$ & $540 \mathrm{MHz}$ \\
Vaseline & $358 \mathrm{MHz}$ & $534 \mathrm{MHz}$ \\
Roger 5880 & $344 \mathrm{MHz}$ & $520 \mathrm{MHz}$ \\
Roger 5870 & $322 \mathrm{MHz}$ & $490 \mathrm{MHz}$ \\
CEM & $218 \mathrm{MHz}$ & $285 \mathrm{MHz}$ \\
Beryllia & $155 \mathrm{MHz}$ & $239 \mathrm{MHz}$ \\
Alumina (95\%) & $110 \mathrm{MHz}$ & $167 \mathrm{MHz}$ \\
Silicon & $89 \mathrm{MHz}$ & $131 \mathrm{MHz}$ \\
Gallium Arsenide & $84 \mathrm{MHz}$ & $126 \mathrm{MHz}$ \\
\hline
\end{tabular}




\section{Conclusion}

Analysis of lumped component representation for reflectarrays designed at $10 \mathrm{GHz}$ constructed above different materials is presented. The effect on the reflection loss and bandwidth performance is discussed in terms of lumped components. The reflection loss and reflection phase curves for reflectarrays with different materials have been obtained using equivalent circuit analysis. It has been shown that Teflon which has the least value of $\varepsilon_{\mathrm{r}}=2.08$ and very low value of $\tan \delta=0.0004$ shows minimum reflection loss $\mathrm{Rl}=0.179 \mathrm{~dB}$ and maximum $10 \%$ and $20 \%$ bandwidths of $360 \mathrm{MHz}$ and $540 \mathrm{MHz}$ respectively. However CEM which has the highest loss tangent value $\tan \delta=0.025$, demonstrates a reflection loss of $\mathrm{Rl}=6.875 \mathrm{~dB}$ offering an improved bandwidth performance than Gallium Arsenide due to lower permittivity value.

\section{Acknowledgment}

This research is fully sponsored by Fundamental Research Grant Scheme (FRGS), Ministry of Higher Education Malaysia (VOT0558). We would like to the staffs of Wireless and Radio Science Center (WARAS) and Radio Communications and Antenna Design Laboratory (RACAD) of Universiti Tun Hussein Onn Malaysia (UTHM) for providing the technical support.

\section{References}

[1] David M. Pozar, D. Targonski, and H. D Syrigos, "Design of Millimeter Wave Microstrip Reflectarrays", IEEE Trans. Antennas and Propagation, vol. 45, No. 2, February 1997.

[2] R. D javor, X. D. Wu, K. chang, "Design and Performane of Microstrip Reflectarray Antenna", IEEE Trans. Antennas and Propagation, vol. 43, No. 9 pp. 932-938,sep 1995.

[3] S. D. Targonski and D. M. Pozar, "Analysis and Design of a Microstrip ReflectarrayUsing Patches of Variable Size," IEEE AP-S/URSI Symposium, Seattle, Washington, pp. 1820-1823, June 1994.

[4] J. Huang and R. J. Pogorzelski,"macrostrip reflectarray with elements having variable rotation angle", IEEE AP-S Symposium Digest, pp. 1280-1283 April 1993.

[5] J. Huang, "Analysis of microstrip reflectarray antenna for microspacecraft applications", Spacecraft Telecommunications Equipment section, TDA Progress report 42-120, February 15,1995.

[6] M. Y. Ismail and M. Inam, "Analysis of Design Optimization of Bandwidth and Loss Performance of Reflectarray Antennas Based on Material Properties", Modern Applied Sci. J. CCSE., vol. 4 No. 1 pp 28-35, Jan. 2010.

[7] M. Bozzi, S. Germani, L. Perregrini, "A Figure of Merit for Losses in Printed Reflectarray Elements", IEEE Antennas and Wireless propogation letters, vol. 3, 2004.

[8] Gary L. Johnson, Solid State Tesla Coil, Kansas State University Press. Manhattan, Kansas, pp. 3_1-3_24, December 2001.

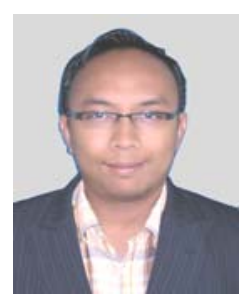

Muhammad Yusof Ismail received B.Eng (Hons) in Electrical \& Electronics Engineering from Universiti Kebangsaan Malaysia in 2002 and $\mathrm{PhD}$ degree in Communications Engineering from Queen's University of Belfast, Northern Ireland, United Kingdom in 2007. Dr. Yusof is currently a Senior Lecturer at Department of Communications Engineering, Universiti Tun Hussein Onn Malaysia. He heads Radio Communications and Antenna Design Laboratory (RACAD). His research interests include design of reconfigurable antennas, frequency selective surfaces, planar microwave absorber and radar cross section control based on active devices. He is presently involved as a project leader as well as research member in different research grant projects awarded by Ministry of Higher Learning Education (MOHE). 


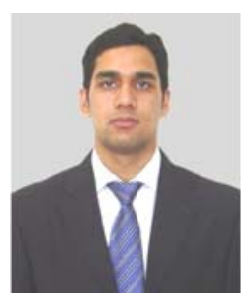

Muhammad Inam Abbasi completed his BSC in Electrical Engineering with major in Telecommunication in 2008 from Centre for Advanced Studies in Engineering (CASE Islamabad), UET, Taxilla, Pakistan. He is currently pursuing his Masters by research in Electrical Engineering from Universiti Tun Hussein Onn Malaysia (UTHM), Batu Pahat, Malaysia. He is also working as a Graduate Research Assistant under the FRGS, Malaysian Higher Education. His research interests include design of planar antennas and reconfigurable reflectarray antennas.

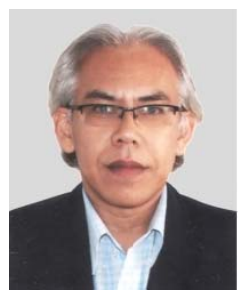

Ahmad Faizal Mohd. Zain completed his Electronic Engineering (Hons) in 1979 and Masters of Engineering in Microwave Communication in 1981 from Sheffield University, United Kingdom. After three years of service as a lecturer at Universiti Kebangsaan Malaysia (UKM), he went to do his doctoral studies at The Pennsylvania State University, United States of America, investigating propagation of extremely low frequency electromagnetic waves generated by modification of the polar ionosphere where he was conferred the Ph.D. in Electrical Engineering in 1990. He returned to UKM and was promoted as an Associate Professor in 1992. He was the Head of the Electrical, Electronic, and System Engineering Department at UKM before transferring to Universiti Tun Hussein Onn Malaysia in 2001 as Professor and Dean of Engineering. He was appointed the Dean of the Centre for Graduate Studies from 2004 to 2008. Prof. Zain is a Fellow of the Institution of Engineers Malaysia, a Fellow of the Academy of Sciences Malaysia, an Associate Senior Fellow of the Institute of Space Science, UKM, and a Senior Member of IEEE. He is also a registered professional engineer in Malaysia. His main research interests are in the characterization of the equatorial ionosphere, antenna and wave propagation, and spectrum management. He established and founded the Centre for Wireless and Radio Science (WARAS) at Universiti Tun Hussein Onn Malaysia. WARAS operates the first and currently the only digital interferometric ionosonde in Malaysia. Prof. Zain now heads the Centre for WARAS.

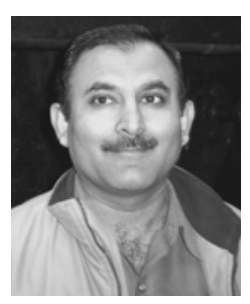

Muhammad Amin received the Bachelor of Engineering (B.E.) degree in avionics from the College of Aeronautical Engineering, NED University, Karachi, Pakistan, in 1988, the Masters degree in electrical engineering with specialization in high frequency techniques from Ruhr University, Bochum, Germany, in 1998, and the Ph.D. degree in antennas for satellite and land communications from The Queen's University Belfast (QUB), Belfast, U.K., in 2006. He taught as an Assistant Professor at the College of Electrical and Mechanical Engineering, National University of Sciences and Technology, Rawalpindi, Pakistan, from 1998 to 2002. He worked as a consultant with TDK Electronics to develop phased array antenna for automotive collision avoidance radar. He was a Research Fellow at The Queens University of Belfast (QUB), Belfast, U.K., for approximately one year and worked an Associate Professor at The Institute Of Space Technology, Islamabad, Pakistan from Oct 2007 to Oct 2009. Since Oct 2009 he is working in Satellite and Upper Atmospheric Research Corporation (SUPARCO) as Head Antenna and EMI/EMC labs at Satellite Research development Centre, Lahore (SRDC-L). His research interests include the development of antennas for radar and cellular communication systems, novel techniques for modulation and RCS reduction. He is inventor of lowest profile dual polarized antenna. He is mentioned in "Marquis Who is Who in the World" 2008 edition published from USA. 


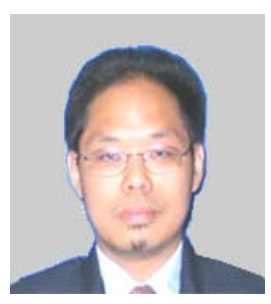

Mohammad Faiz Liew Abdullah received BSc (Hons) in Electrical Engineering (Communication) in 1997, Dip Education in 1999 and MEng by research in Optical Fiber Communication in 2000 from University of Technology Malaysia (UTM). He completed his PhD in August 2007 from The University of Warwick, United Kingdom in Wireless Optical Communication Engineering. He started his career as a lecturer at Polytechnic Seberang Prai (PSP) in 1999 and was transferred to UTHM in 2000 (formerly known as PLSP). At present he is a senior lecturer in the Department of Communication Engineering, Faculty of Electrical \& Electronic Engineering, University Tun Hussein Onn Malaysia (UTHM). He had 10 years experience of teaching in higher education, which involved the subject Optical Fiber Communication, Advanced Optical Communication, Advanced Digital Signal Processing and etc. His research area of interest are wireless and optical communication and robotic in communication. 